\title{
Pernicious Anemia Presenting With Pancytopenia and Hemolysis: A Case Report
}

\author{
Eric Gladstone
}

\begin{abstract}
Vitamin B12 deficiency is a well-known cause of anemia. Pancytopenia and hemolysis are less frequently observed in vitamin B12 deficiency. Reported is the case of a 58-year-old man who presented with dyspnea on exertion, fatigue, and unintentional weight loss. $\mathrm{He}$ was found to have severe anemia (hemoglobin $3.3 \mathrm{~g} / \mathrm{dL}$ ) in association with pancytopenia and hemolysis. Extensive workup revealed vitamin B12 deficiency secondary to pernicious anemia. A literature review confirmed that this case demonstrates the rare occurrence of severe anemia in association with pancytopenia and hemolysis due to B12 deficiency.
\end{abstract}

Keywords: Pernicious; Anemia; Pancytopenia; B12; Hemolysis

\section{Introduction}

Vitamin B12 deficiency is a common cause of anemia. Pernicious anemia leading to pancytopenia and hemolysis is rare. Providers should be cognizant of this phenomenon as the workup of pancytopenia and hemolysis can be invasive and costly.

\section{Case Report}

A 58-year-old African American male presented to the emergency room (ER) with 6 weeks of progressively worsening dyspnea on exertion, fatigue and unintentional weight loss. He had a past medical history of iron deficiency anemia, gout, and osteoarthritis. He denied other respiratory symptoms. He never used tobacco. He reported frequent gum bleeding when brushing his teeth but denied nosebleeds, hemoptysis, hematochezia, melena or hematuria. He reported intermittent chills and a 15-pound weight loss which he attributed to poor appetite. Review of systems was otherwise unremarkable. He ate a balanced diet and was not a vegetarian or vegan. He recalled

Manuscript submitted February 8, 2019, accepted February 25, 2019

Department of Medicine, Lenox Hill Hospital, New York, NY, USA. Email: egladstone@northwell.edu

doi: https://doi.org/10.14740/jmc3269 being diagnosed with iron deficiency anemia at an ER 5 year prior, requiring transfusion at the time of diagnosis. He did not recall any workup for the cause of his anemia. He did not have a primary care physician. Since then, he had been taking iron supplements twice a week. His only other medication was naproxen, which he took only sparingly on occasion. He sometimes smoked marijuana for recreation, but denied alcohol use. He was recently divorced and was sexually active with a few new female partners, using condoms consistently. His father developed prostate carcinoma at around the age of 70 . There was no other known family history of malignancy or rheumatologic disorders.

At presentation, his vital signs were stable. Exam revealed subconjunctival pallor, two mildly enlarged submandibular lymph nodes that were mobile and nontender, hepatomegaly with right upper quadrant tenderness, scattered ecchymoses of the lower extremities and hyperpigmentation of the gums and interphalangeal joints. Labwork revealed hemoglobin of $3.3 \mathrm{~g} /$ $\mathrm{dL}$, hematocrit 9.4\%, mean corpuscular volume (MCV) 119 fL, red cell distribution width (RDW) $26 \%$, white blood cell (WBC) $3,000 / \mu \mathrm{L}$ (55\% lymphocytes, $42 \%$ neutrophils, $2 \%$ eosinophils, $1 \%$ basophils), platelets $41,000 / \mu \mathrm{L}$, and reticulocyte percent 1.4 (reticulocyte index 0.13). Manual smear showed macrocytosis, hypersegmented neutrophils, large platelets, and many atypical lymphocytes as well as occasional schistocytes. Prothrombin time (PT) was $13.1 \mathrm{~s}$, partial thromboplastin time (PTT) 28.4 s, fibrinogen 269 mg/dL, D-dimer 573 ng/mL, lactate dehydrogenase $(\mathrm{LDH})>2,500 \mathrm{U} / \mathrm{L}$, and haptoglobin < $10 \mathrm{mg} / \mathrm{dL}$. Comprehensive metabolic panel (CMP) was unremarkable. Urinalysis (UA) showed small bilirubin, urobilinogen $>8 \mathrm{EU} / \mathrm{dL}$, trace leukocyte esterase and trace blood. Chest $\mathrm{X}$-ray (CXR) was unremarkable. Computed tomography (CT) abdomen and pelvis with intravenous (IV) contrast showed hepatomegaly (right lobe spanning $19 \mathrm{~cm}$ ) and several small hepatic hypodensities too small to be characterized, but no splenomegaly, lymphadenopathy or other evidence of malignancy. He was transfused four units of packed red blood cells and was admitted for further workup. Serum vitamin B12 level was low at $<150 \mathrm{pg} / \mathrm{mL}$. Folate level was normal at $13.7 \mathrm{ng} / \mathrm{mL}$. Iron studies showed elevated total serum iron, ferritin, and percentage of saturation with low total iron binding capacity (TIBC). Human immunodeficiency virus (HIV) and hepatitis C screens were negative. Epstein-Barr virus (EBV) serologies were consistent with prior exposure. Antinuclear antibody (ANA) titer was 1:160. Quantitative rheumatoid factor was $9 \mathrm{IU} / \mathrm{mL}$ (WNL). Transglutaminase antibodies were negative. Positron emission tomography (PET) CT showed no evidence of ma- 
lignancy. A bone marrow biopsy was performed. The marrow showed myeloid and erythroid hyperplasia with megaloblastic changes of erythroid elements. Cytogenetic analysis showed no consistent abnormalities and no evidence of leukemia or lymphoma. Lab findings conclusive for pernicious anemia returned on hospital day 3 ; these included elevated levels of homocysteine $(155 \mathrm{~mol} / \mathrm{L})$ and MMA $(88,883 \mathrm{nmol} / \mathrm{L})$ and the detection of intrinsic factor antibodies.

\section{Discussion}

Vitamin B12, also termed cobalamin, is required for formation of hematopoietic cells. Cobalamin is a cofactor used in the synthesis of DNA as well as in metabolism of fatty acids and amino acids. Additionally, through an incompletely known mechanism, vitamin B12 is used in neuronal myelination.

Pancytopenia is a rare manifestation of vitamin B12 deficiency. More commonly, simple anemia is observed. The differential for pancytopenia is broad, including infection, autoimmune conditions, marrow failure, and marrow space-occupying lesions. Pancytopenia due to vitamin B12 deficiency is more common in developing countries [1]. Pancytopenia occurs in cases of severe vitamin B12 deficits that are sustained over time. The mechanism of the cytopenias is defective DNA synthesis [2]. While all hematopoietic cell lines are affected, the impact on erythrocytes is greatest. In one study of 201 patients with documented cobalamin deficiency, two-thirds of patients showed hematologic abnormalities [3]. Macrocytosis was the most commonly observed finding (54\%), followed by anemia (37\%), hypersegmented neutrophils (32\%), leukopenia $(13.9 \%)$, and thrombocytopenia (9.9\%) [3]. Pancytopenia was seen in $5 \%$ of patients and hemolytic anemia in $1.5 \%$ [3]. Thrombocytopenia is usually due to ineffective platelet production. While some patients have marrow aplasia, megakaryocytic mass and platelet survival are usually normal $[4,5]$. There are cases in the literature of isolated thrombocytopenia; however this is rare [6]. Leukopoietic cells may reach large dimensions and have trouble crossing marrow sinuses to reach the peripheral blood, contributing to leukopenia [7]. Only rarely does the thrombocytopenia and leukopenia cause clinical problems.

Cobalamin deficiency can lead to both intramedullary and extramedullary hemolysis. Intramedullary hemolysis is more common and occurs due to macrophage destruction of megaloblastic cells in the bone marrow. Conversely, extramedullary hemolysis in cobalamin deficiency ("pseudothrombotic microangiopathy") occurs due to fragmentation of erythrocytes in capillaries. This appears to be multifactorial. Cobalamin deficiency leads to decreased deformability of the red blood cell (RBC) membrane as well hyperhomocysteinemia promoting endothelial dysfunction [8-10]. In both intramedullary and extramedullary hemolysis, serum LDH levels will be high while haptoglobin levels will be low. In purely intramedullary hemolysis, schistocytes would not be expected on peripheral smear. When schistocytes are seen, other types of microangiopathic hemolytic anemia, most importantly thrombotic thrombocytopenic purpura (TTP), should be considered. TTP is a syndrome of thrombocytopenia, hemolytic anemia, kidney injury, fever and changes in mental status. Distinguishing TTP from pseudothrombotic microangiopathy based on routine labs is imperative as ADAMTS13 assay results are not rapidly available. In pseudothrombotic microangiopathy, the reticulocyte count should be low. Bilirubin levels will be lower than expected as destruction of immature erythroblasts releases less hemoglobin than with mature erythrocytes. Alternatively, LDH levels and platelets are generally lower in TTP.

Animal products are the main source of cobalamin. Strict vegetarians are at greater risk of deficiency. Other causes of deficiency include pernicious anemia, gastritis and impaired absorption. Impaired absorption is often secondary to inflammatory bowel disease (IBD), surgery, pancreatic insufficiency and celiac disease. Bacterial overgrowth and infection with fish tapeworm are also predisposing factors. Common medications such as metformin, proton pump inhibitors and histamine receptor antagonists can interfere with cobalamin absorption. Rarely, transcobalamin deficiency (an autosomal recessive genetic disorder) is the culprit. The most common cause of deficiency is pernicious anemia. Pernicious anemia is caused by autoantibodies targeting intrinsic factor or gastric parietal cells.

Stores of vitamin B12 are generally large enough to supply adequate levels for over 5 years. Aside from anemia, signs of deficiency include glossitis, neuropathies and cognitive dysfunction. Degeneration of the posterior and lateral columns of the spinal cord in cobalamin deficiency is termed subacute combined degeneration. Classically, the neuropathy manifests as symmetric paresthesias, numbness or problems with ambulation. The legs are affected before the arms.

Diagnosing deficiency usually begins with testing of the serum vitamin B12 level. The Schilling test is not routinely used anymore. Confirmation of deficiency should be performed by measuring levels of homocysteine and methylmalonic acid. Both will be elevated in vitamin B12 deficiency, as opposed to folate deficiency where MMA will be normal. The CBC may show an elevated mean corpuscular volume. On a peripheral smear one may observe hypersegmented neutrophils. Hypersegmented neutrophils possess $\geq$ five lobes. A bone marrow evaluation is likely to reveal a markedly hypercellular marrow with megaloblastic erythroid hyperplasia, giant metamyelocytes, and frequent mitoses.

In this case, the patient presented with many classical features of vitamin B12 deficiency. The best initial clue was his MCV of $119 \mathrm{fL}$. While vitamin B12 deficiency does not always present with an elevated MCV, an MCV of $>120 \mathrm{fL}$ is $99 \%$ specific for vitamin B12 deficiency [11]. Other causes of macrocytosis such as heavy alcohol use, folate deficiency, and reticulocytosis were excluded. The finding of hypersegmented neutrophils in addition to MCV $>100 \mathrm{fL}$ has a specificity of $97 \%$ for vitamin B12 deficiency [11]. Although vitamin B12 deficiency can account for pancytopenia, we decided to perform a bone marrow biopsy and PET-CT to look for evidence of malignancy. The patient's history of unintentional weight loss was one driving factor. Interestingly, vitamin B12 deficiency has been associated with constitutional symptoms including fevers [12]. The patient's elevated LDH and low haptoglobin likely reflected a primarily intramedullary hemolysis 
as few schistocytes were observed.

\section{Acknowledgments}

None to declare.

\section{Financial Disclosure}

None to declare.

\section{Conflict of Interest}

There is no financial support or relationships that may pose conflict of interest.

\section{Informed Consent}

Written consent was obtained from the patient.

\section{Author Contributions}

EG was involved in patient care and writing the manuscript.

\section{References}

1. Premkumar M, Gupta N, Singh T, et al. Cobalamin and folic acid status in relation to the etiopathogenesis of pancytopenia in adults at a tertiary care centre in North India. Anemia. 2012;2012:707402.
2. Wadood Khan ZA, Vidyasagar S, Bekur R, Belurkars S, et al. Subhyaloid haemorrhage in a patient with vitamin B12 deficiency: a unique presentation. J Clin Sci Res. 2013;2:161-164.

3. Andres E, Affenberger S, Zimmer J, Vinzio S, Grosu D, Pistol G, Maloisel F, et al. Current hematological findings in cobalamin deficiency. A study of 201 consecutive patients with documented cobalamin deficiency. Clin Lab Haematol. 2006;28(1):50-56.

4. Edited by McKrae. Thrombocytopenia. 2006.

5. Ghosh K, Sarade R, Varma N, et al. Megakaryocytic thrombocytopenia of nutritional B12 deficiency. Trop Geogr Med. 1988;40(2):158-160.

6. Okur M, Ozkan A, Gunes C, Kaya M, Kocabay K. Case of isolated thrombocytopenia due to cobalamin deficiency. Int J Hematol. 2011;94(5):488-490.

7. Briani C, Dalla Torre C, Citton V, Manara R, Pompanin S, Binotto G, Adami F. Cobalamin deficiency: clinical picture and radiological findings. Nutrients. 2013;5(11):4521-4539.

8. Malla M, Seetharam M. To treat or not to treat: a rare case of pseudo-thrombotic thrombocytopenia purpura in a Jehova's Witness. Transfusion. 2016;56(1):160-163.

9. Tuten N, Bennett C, Babcock W. Thrombotic thrombocytopenic purpura or cobalamin deficiency? A case report and review. Clin Case Rep Rev. 2015;1(8):157-159.

10. Acharya U, Gau JT, Horvath W, Ventura P, Hsueh CT, Carlsen W. Hemolysis and hyperhomocysteinemia caused by cobalamin deficiency: three case reports and review of the literature. J Hematol Oncol. 2008;1:26.

11. Savage DG, Ogundipe A, Allen RH, Stabler SP, Lindenbaum J. Etiology and diagnostic evaluation of macrocytosis. Am J Med Sci. 2000;319(6):343-352.

12. Manuel K, Padhi S, G'Boy Varghese R. Pyrexia in a patient with megaloblastic anemia: a case report and literature review. Iran J Med Sci. 2013;38(2 Suppl):198-201. 